|| Print ISSN: 2589-7837 || Online ISSN: 2581-3935 ||

International Journal of Medical Science and Diagnosis Research (IJMSDR)

Available Online at www.ijmsdr.com

NLM (National Library of Medicine ID: 101738824)

\title{
A CASE REPORT OF ELIMINATION OF ALVEOLAR DEFECTS BY SECONDARY BONE GRAFTING.
} Dr Charan Teja Vemagiri ${ }^{1}$, Dr Damera Srikanth ${ }^{2}$, Dr Chandrababu $V^{3}$, Dr Siva Ganesh ${ }^{4}$

${ }^{1}$ Assistant Professor, Department of Paediatric and Preventive Dentistry, GSL Dental College and Hospital.

${ }^{2}$ Reader, Department of Oral and Maxillofacial Surgery, GSL Dental College and Hospital.

${ }^{3}$ Reader, Department of Oral and Maxillofacial Surgery, GSL Dental College and Hospital.

${ }^{4}$ Assistant Professor, Department of Oral and Maxillofacial Surgery, GSL Dental College and Hospital.

Conflicts of Interest: Nil

Corresponding author: Dr Charan Teja Vemagiri

DOI: https://doi.org/10.32553/ijmsdr.v4i9.665

\section{Abstract}

The secondary alveolar bone grafting is an integral component of contemporary rehabilitation of the patients with cleft lip and palate with alveolar defects. Iliac bone graft is frequent secondary graft used in the correction of alveolar defects. There is successful rehabilitation of osseous component post operatively.

\section{Introduction:}

Among the many congenital birth defects, there have been more than 250 different types of facial clefting disorders described in the literature. The overall incidence of cleft lip and palate is at 1:500 live births in Asians and 1:750 in the United States. Out of the various combinations of isolated cleft lip, isolated cleft palate, and bi lateral cleft lip and palate, the unilateral cleft lip and palate is the most common in occurrence. In $75 \%$ of the cleft lip and palate occurrences, the cleft runs through the alveolar ridge. ${ }^{1}$

Although exact etiology is still obscure, it is the result of multifactorial genetic and environmental factors. The environmental factor includes nutritional deficiencies. Maternal diabetes, smoking, radiation, viruses, drugs etc do influence. Embryological basis has been cited for this deformity.

Alveolar cleft is the least of the deformity associated with cleft palate and is always seen in combined cleft lip and palate. This can be unilateral or bilateral, an isolated one or a complete one extending posteriorly. It is due to the failure of fusion between the premaxilla and the lateral process of the maxilla.

The initial attempts at the treatment of alveolar cleft defects date back to 1901, when Von Eiselberg used pedicled bone to fill an alveolar defect. ${ }^{2}$

Although a number of autogenous and allogenic materials are used in maxillofacial reconstruction, autogenous grafts are preferred in alveolar clefts. Though there are varieties of autogenous graft materials like iliac crest bone, tibia, rib, mandibular symphysis, calvarium etc, the preferred one is cancellous marrow of ilium.

The primary choice of bone graft is autogenous cancellous marrow of ilium since a viable amount of cancellous bone can be obtained from this site. Concerns related to the anatomic site have been that iliac crest is one of the growth centres of the ilium and surgical intervention could produce undesirable alteration in hip and the morbidity of the procedure. With the refinement in the techniques and surgical experience much of the complications can be avoided.

\section{Case report}

A 12-year-old female presented with a complain of difficulty in speech and regurgitation of fluids when taken from nose. The intraoral picture shows deficiency in the left canine region. The radiographic picture shows radiolucency in the alveolus at the canine region. ${ }^{3}$

The intra oral approach is planned under general anaesthesia, the incision is placed to expose the alveolar defect and simultaneously the graft is harvested from the iliac crest region. The corticocancellous graft is crushed and mixed with autologous blood and placed at the intra oral donor site. Post-operative picture shows that the healing is satisfactory and four months post-operative radiograph shows radioopacity with adequate amount of bone formation.

\section{Pre-operative clinical pictures:}
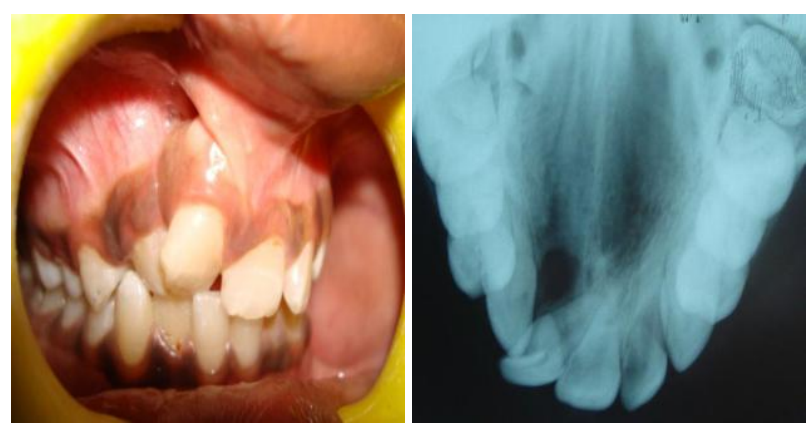


\section{Post-operative clinical pictures}

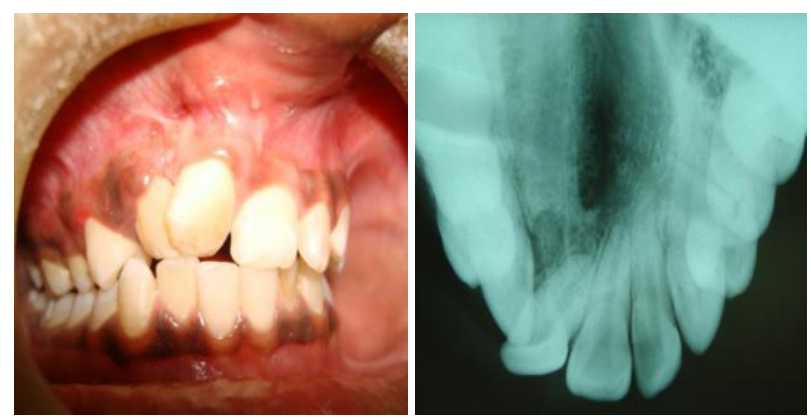

Discussion:

The initial attempts for the treatment of alveolar cleft dates back to 1901, when Von Eiselberg used pedicled bone to fill alveolar defect. ${ }^{2}$ since that time, the complex management of cleft alveolus has advanced tremendously. In maxillofacial surgery, autologous bone grafting is used for treatment of bone defects attributable to tumors, trauma or age related atrophy of the jaw is now a standard procedure. The first documented bone transplant was performed by the Flemish surgeon Job Van Meekeren [1611-1666] who first described the successful transplant of a piece of skull from a dog into a defect in a human skull. $^{3}$

Autologous bone grafting for patients with cleft lip/palate has been practiced for many decades. Early attempts to fill in the bone defect used rib struts at the time of lip repairso called 'primary bone grafting'. The results were generally poor and this practice is now obsolete. ${ }^{4}$ Secondary alveolar bone grafting was introduced in 1972 by Boyne and Sands. ${ }^{5}$

Fresh autogenous cancellous bone is the ideal bone-graft material for SABG because it supplies living, immune compatible bone cells essential to osteogenesis and becomes fully integrated with maxilla. Various donor sites for autogenous cancellous bone are iliac crest, calvarium, mandibular symphysis and tibia. ${ }^{6}$

The iliac crest remains the gold standard for SABG because of its accessibility, abundance of cancellous bone, relative ease of bone harvest and the fact that the defect is both well covered and not prone to pathological fracture. ${ }^{9}$

However, the main disadvantage with iliac crest harvest is reported to be the discomfort that results in delayed ambulation and prolonged hospitalization. ${ }^{7}$ Even with iliac bone, insufficient osteo-regeneration may occur due to several factors like patients age, cleft width, influence of functional stress; so platelet- rich-plasma [PRP] extracted from autologous whole blood known to have a number of different growth factors in high concentration is known to produce successful results when used for alveolar bone grafting. ${ }^{8}$

\section{Conclusion:}

Bone substitutes like tissue engineered bone and recombinant human one morphogenetic protein-2[rh BMP-2] are being used to augment the alveolar ridge and fill its defects. However, much more research and development is necessary to study their properties and further randomized clinical trials are needed before they can be a substitute for autogenous bone. This aspect of translational research can change and improve the repair of cleft alveolus as these procedures are less invasive for the patients.

\section{References:}

1. Amin kazemi, Jeffrey W. Stearns and Raymond J Fonseca.Secondary grafting in the alveolar cleft patient. Oral Maxillofacial Surg Clin $\mathrm{N}$ Am 2002; 14: 477-490.

2. Koberg WR. Present view of bone grafting in cleft palate [review].J Maxillofac Surg 1973; 1:185-93.

3. Heidrun Schaaf, Stefan Lendeckel, Hans-peter Howaldt and Philipp Streckbein. Donor site morbidity after bone harvesting from the anterior iliac crest. Oral surg oral med oral pathol oral radiol endod 2010; vol-109:52-58.

4. Alex Habel, Debbie Sell and Michael Mars. Management of cleft lip and palate. Archives of disease in childhood 1996; 74:360-366.

5. McCanny C M and D.P. Roberts-Harry. A comparison of two different bone-harvesting techniques for secondary alveolar bone grafting in patients with cleft lip and palate. Cleft palateCraniofacial Journal, Sept 1998; vol.35, No.5:442-446.

6. Rawashdeh M A. Morbidity of iliac crest donor site following open bone harvesting in cleft lip and palate patients. Int J oral maxillofac surg 2008; 37:223-227.

7. McCanny C M and D.P. Roberts-Harry. A comparison of two different bone-harvesting techniques for secondary alveolar bone grafting in patients with cleft lip and palate. Cleft palateCraniofacial Journal, Sept 1998; vol.35, No.5:442-446.

8. Mitsuyoshi Lino, Hiroaki Ishi Junichi Sato and Kanichi Seto. Histological evaluation of autogenous iliac particulate cancellous bone and marrow grafted to alveolar clefts - A preliminary report of five young adult cases. Cleft palate-Craniofacial Journal, January 2000; vol.37, No.1:55-60.

9. Harald Eufinger and Heikki Leppanen. Iliac crest donor site morbidity following open and closed methods of bone harvest for alveolar cleft osteoplasty. Journal of Cranio-Maxillofacial Surgery 2000; 28:31-38. 